\title{
Growth Factors for Skeletal Muscle Tissue Engineering
}

\author{
Brian C. Syverud $^{\mathrm{a}}$ Keith W. VanDusen ${ }^{\mathrm{b}}$ Lisa M. Larkin ${ }^{\mathrm{a}} \mathrm{b}$ \\ ${ }^{\mathrm{a}}$ Biomedical Engineering and ${ }^{\mathrm{b}}$ Molecular and Integrated Physiology, University of Michigan, Ann Arbor, Mich., USA
}

\section{Key Words}

Growth factors · Skeletal muscle · Tissue engineering

\begin{abstract}
Tissue-engineered skeletal muscle holds promise as a source of graft tissue for repair of volumetric muscle loss and as a model system for pharmaceutical testing. To reach this potential, engineered tissues must advance past the neonatal phenotype that characterizes the current state of the art. In this review, we describe native skeletal muscle development and identify important growth factors controlling this process. By comparing in vivo myogenesis to in vitro satellite cell cultures and tissue engineering approaches, several key similarities and differences that may potentially advance tissueengineered skeletal muscle were identified. In particular, hepatocyte and fibroblast growth factors used to accelerate satellite cell activation and proliferation, followed by addition of insulin-like growth factor as a potent inducer of differentiation, are proven methods for increased myogenesis in engineered muscle. Additionally, we review our recent novel application of dexamethasone (DEX), a glucocorticoid that stimulates myoblast differentiation, in skeletal muscle tissue engineering. Using our established skeletal muscle unit (SMU) fabrication protocol, timing- and dose-dependent effects of DEX were measured. The supplemented SMUs demonstrated advanced sarcomeric structure and significantly increased myotube diameter and myotube fusion
\end{abstract}

compared to untreated controls. Most significantly, these SMUs exhibited a fivefold rise in force production. Thus, we concluded that DEX may serve to improve myogenesis, advance muscle structure, and increase force production in engineered skeletal muscle.

(c) 2016 S. Karger AG, Basel

\section{Introduction}

Proposed applications for tissue-engineered skeletal muscle include implantation as a graft material for repair of traumatic damage [Bach et al., 2003; Koning et al., 2009], recapitulation in vitro of native development and

\section{Abbreviations used in this paper}

DEX dexamethasone

DRG dorsal root ganglion

ECM extracellular matrix

FGF fibroblast growth factor

HGF hepatocyte growth factor

IGF insulin-like growth factor

MHC myosin heavy chain

mTOR mammalian target of rapamycin

PDGF platelet-derived growth factor

Shh sonic hedgehog

SMU skeletal muscle unit

TGF transforming growth factor

\section{KARGER}

(C) 2016 S. Karger AG, Basel

E-Mail karger@karger.com

www.karger.com/cto
Lisa M. Larkin

University of Michigan

2025 BSRB, 109 Zina Pitcher Place

Ann Arbor, MI 48109-2200 (USA)

E-Mail llarkin@umich.edu 
regeneration for detailed physiological study or pharmaceutical testing [Lee and Vandenburgh, 2013], and use as biomechanical actuators [Sakar et al., 2012; Neal et al., 2014]. In all cases, mimicking the complex structure and function of skeletal muscle in vivo is an essential consideration. To date, however, engineered tissues have been characterized by a neonatal phenotype in terms of vascularity, force production, and structural maturity [Williams et al., 2013; Juhas et al., 2014]. Without major advances, especially in the area of vascularization, use of engineered muscle as a graft material will be severely limited. Implantation of engineered skeletal muscle into an in vivo regenerative environment, however, has promoted development towards the adult phenotype [Corona et al., 2014; VanDusen et al., 2014], and several recent studies have attempted to utilize key chemical and mechanical stimuli to improve the maturity of these engineered muscles in vitro [Dennis et al., 2009; Martin et al., 2013]. This review focuses on important in vitro biochemical stimuli, summarizing the current state of the art in growth factors utilized for skeletal muscle tissue engineering. Additionally, data are presented on the steroid dexamethasone (DEX) and its effects on tissue-engineered skeletal muscle units (SMUs) as a novel stimulus for in vitro muscle maturation.

\section{Growth Factors in Native Skeletal Muscle Development and Regeneration}

\section{Skeletal Muscle Development during Embryogenesis}

Before attempting to describe the ideal in vitro biochemical environment for engineered skeletal muscle, it is necessary to understand the in vivo environment tissue engineers seek to emulate. During embryogenesis, morphogen gradients control patterning of the developing tissues. Following germ layer formation in the prepatterned embryo, localized variations in gene expression and signaling gradients prompt condensations of the paraxial mesoderm into somites (fig. 1) [Bentzinger et al., 2012]. Genes in the Notch and Wnt pathways prompt somitogenesis in concert with spatiotemporal gradients of fibroblast growth factor (FGF) and Wnt proteins. The most dorsal section of the somite becomes the dermomyotome, from which the majority of skeletal muscles are derived. Myogenesis is initiated as the Pax3+ progenitor cell pool present in the somite delaminates and progressively establishes the primary myotome [Biressi et al., 2007]. Members of the Wnt family of proteins again play a central role in this process. Through binding to Frizzled receptors, Wnt signaling activates the $\beta$-catenin/T-cell factor complex and induces somite patterning and expression of the myogenic transcription factors Pax 3 and Myf5 [Bentzinger et al., 2012]. A small subset of the progenitor cell pool migrates into the myotome, proliferates, and then terminally differentiates into myoblasts. In turn, the terminally differentiated myoblasts fuse with each other to form the first multinucleated myotubes and primary myofibers [Buckingham and Mayeuf, 2012]. This stage of myogenesis is primarily regulated by the canonical myogenic regulatory factors: Myf5, MyoD, and myogenin. Sonic hedgehog (Shh), released from the notochord and floor plate of the neural tube, promotes Myf5 expression and commitment to the myogenic lineage [Bentzinger et al., 2012; Buckingham and Mayeuf, 2012]. With the establishment of innervation, the remaining cells from the progenitor pool differentiate into myoblasts and fuse to form secondary myofibers. In addition, a subset of skeletal muscle progenitor cells start to coexpress Pax3 and Pax7, becoming postnatal progenitor cells often referred to as satellite cells [Mauro, 1961; Grefte et al., 2007]. These satellite cells are not activated during embryonic myogenesis and remain as a reserve pool for postnatal muscle growth and regeneration. Bone morphogenic proteins, a subclass of the transforming growth factor- $\beta$ (TGF $\beta$ ) superfamily, serve to preserve this progenitor pool by inhibiting Myf5 and MyoD expression while upregulating Pax3.

In summary, myogenesis during embryonic development is primarily regulated by $\mathrm{Myf} 5, \mathrm{MyoD}$, and myogenin [Buckingham and Mayeuf, 2012], but several molecular signals and growth factors interact with these myogenic regulatory factors. Wnt and FGF gradients direct initial somitogenesis. Subsequently, Shh and Wnt signaling lead to specification and expression of Myf5. Additionally, TGF $\beta$ is known to act through serine-threonine kinase receptors, activating SMAD proteins inhibiting Myf5 and MyoD induction. As a result, the onset of skeletal muscle formation is delayed and the myogenic progenitor pool is preserved. Finally, FGF acts as an antagonist to TGF $\beta$ in regulating the equilibrium between renewal and differentiation of the progenitor pool. Specifically, FGF upregulates Myf5 and MyoD, promotes ac-

Fig. 1. Initial skeletal muscle formation during embryogenesis. a Development of the somite and subsequent establishment of the myotome, distal to the dorsal root ganglion (DRG) and the neural tube. The initial myogenic lineage of the progenitor cells involved is detailed on the left. $\mathbf{b}$ Regulation of progenitor cell renewal and differentiation by myogenic regulatory factors and external signaling. Adapted from Buckingham and Mayeuf [2012].

(For figure see next page.) 


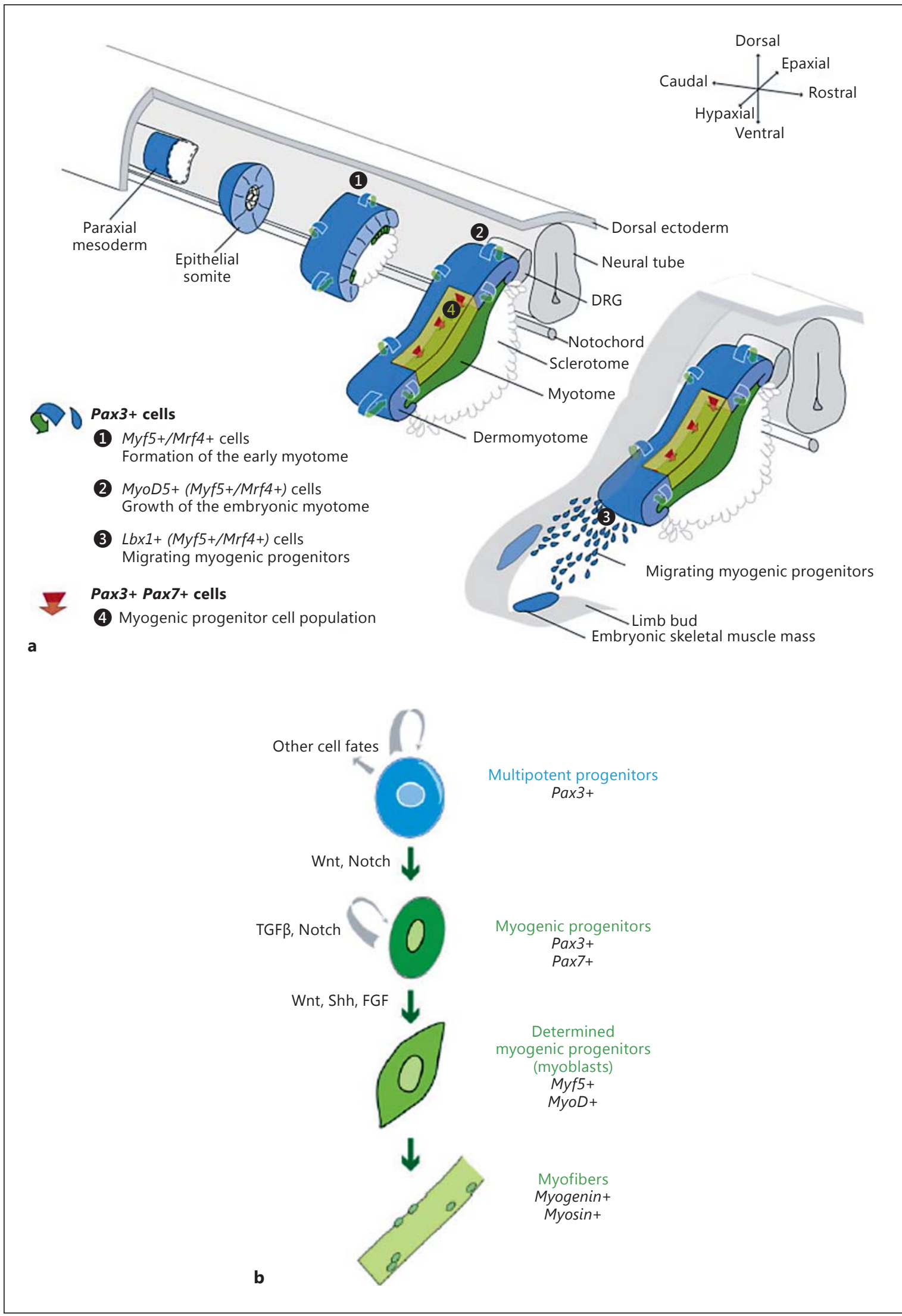

Growth Factors for Skeletal Muscle Tissue 
Fig. 2. Growth factor signaling in skeletal muscle regeneration. The microenvironment surrounding the satellite cell (SC) niche plays a key role in the repair of skeletal muscle damage. Resident immune cells, ECM, and capillary and neural networks compose this niche. This schematic describes a simplified process by which essential growth factors (FGF, TGF $\beta$, IGF, and PDGF) are supplied to stimulate and regulate SCs.

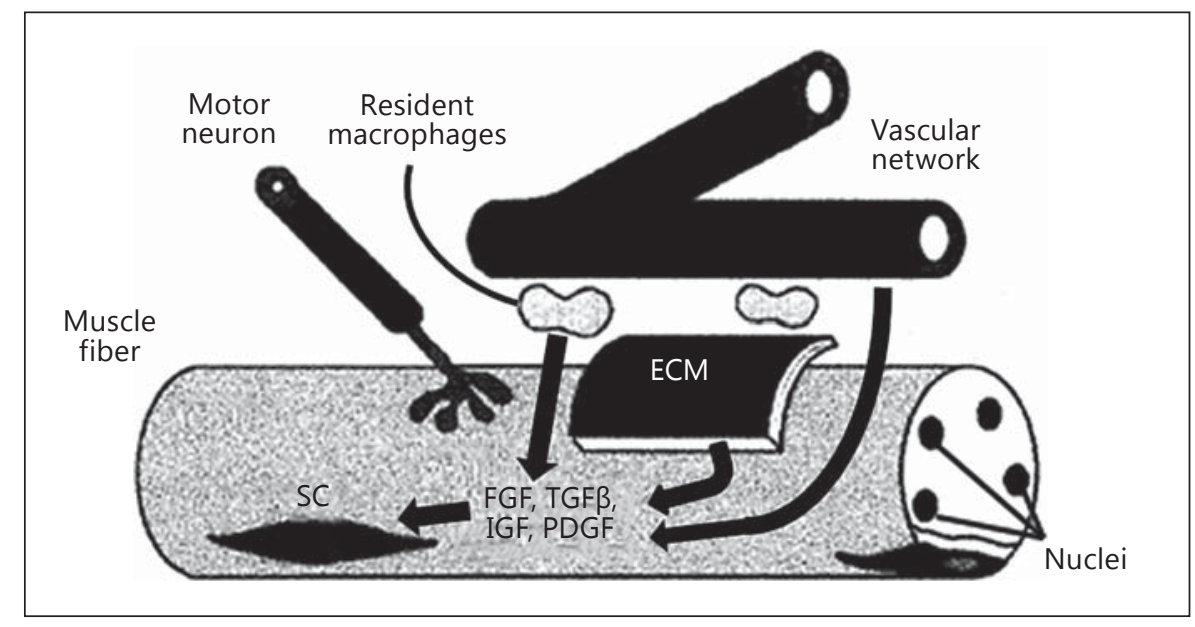

tivation of progenitor cells in the myogenic lineage [Schiaffino and Mammucari, 2011], and begins the transition to postnatal skeletal muscle development.

\section{Postnatal Skeletal Muscle Development}

Postnatal skeletal maturation and lengthening of skeletal muscle relies on the contribution of nuclei and contractile proteins by satellite cells to keep pace with the growing skeleton and to mature in structure. This process is characterized by the addition of new sarcomeres along the length of each fiber, establishment of myotendinous junctions, and the transition in myosin heavy chain (MHC) expression from embryonic to adult fast and slow isoforms [Dhawan and Rando, 2005; Biressi et al., 2007]. Satellite cells provide the nuclei required to regulate this continued growth. Until activated, satellite cells remain quiescent under the basal lamina of skeletal muscle fibers and are characterized by the expression of Pax7 [Mauro, 1961]. The surrounding stem cell microenvironment or niche, composed of extracellular matrix (ECM), vascular and neural networks, neighboring cells, and growth factors, is highly influential on myogenic function (fig. 2) [Cosgrove et al., 2009; Yin et al., 2013]. In response to a complex series of signals, satellite cells are activated and progress toward a committed myogenic lineage, with a subpopulation returning to quiescence to maintain the progenitor pool. As in embryonic development, Notch signaling and Wnt proteins act as essential regulators of postnatal maturation of the satellite cell. In particular, Wnt3a signaling promotes satellite cell activation and differentiation, whereas Wnt7a induces self-renewal and maintenance of the satellite cell pool [Bentzinger et al., 2012]. Mammalian (or mechanistic) target of rapamycin
(mTOR) also plays a key role in mediating postnatal satellite cell activation, proliferation, and differentiation. Although more commonly associated with skeletal muscle hypertrophy and homeostasis, mTOR has recently been shown to regulate satellite cell activity and myogenesis by upregulating expression of Pax7, Myf5, MyoD, and myogenin [Zhang et al., 2015]. Additionally, several growth factors stimulate quiescent satellite cells. Hepatocyte growth factor (HGF) is present in inactive form in the ECM adjacent to satellite cells [Dhawan and Rando, 2005]. When released due to injury or length damage, HGF is thought to bind c-met receptors present on quiescent satellite cells, leading to their activation. Similarly, members of the FGF family are present in the satellite cell niche and bind to quiescent satellite cell receptors following FGF release [Dhawan and Rando, 2005; Buckingham and Mayeuf, 2012]. After activation via growth factors such as HGF or FGF, those satellite cells induced to a myogenic lineage are often referred to as myogenic precursor cells or myoblasts, characterized by their expression of canonical myogenic transcription factors MyoD and Myf5. Following their proliferation and differentiation, the myoblasts fuse with maturing muscle fibers and promote protein synthesis and muscle growth. Connective tissue fibroblasts in the ECM have been shown to interact with satellite cells throughout this stage, with reciprocal signaling between the two cell types prompting increased proliferation of both [Murphy et al., 2011]. Furthermore, expression of the transcription factor Tcf 4 by connective tissue fibroblasts intrinsically regulates the maturation of $\mathrm{MHC}$ isoforms through $\beta$-catenin activation [Mathew et al., 2011; Buckingham and Mayeuf, 2012]. In conclusion, postnatal skeletal muscle develop- 
ment depends on the activation and myogenic differentiation of satellite cells. Although the satellite cell pool is considered heterogeneous, several common signaling pathways play essential roles in this myogenic lineage, with HGF and FGF as the primary growth factors involved.

\section{Skeletal Muscle Homeostasis}

Once skeletal maturation is complete, skeletal muscle homeostasis is maintained through hypertrophy and atrophy. Hypertrophy and regeneration are primarily regulated by a signaling pathway initiated by insulin-like growth factor (IGF)-1 [Schiaffino and Mammucari, 2011]. Whether expressed by muscle cells in response to injury and exercise, secreted by macrophages and endothelial cells with inflammation, or supplied by the circulatory system in the blood, IGF levels increase rapidly in preparation for protein synthesis [Zanou and Gailly, 2013]. Following IGF-1 binding, an intracellular cascade mediates its effects (fig. 3) [Glass, 2003, 2005]. Among these intracellular signals, Akt, or protein kinase B, plays an essential role [Jackman and Kandarian, 2004]. To promote protein synthesis and hypertrophy, Akt indirectly activates mTOR while simultaneously inhibiting glycogen synthase kinase (GSK3 $\beta$ ). At the same time, Akt prevents protein degradation and muscle atrophy by blocking the FoxO family of transcriptions factors [Sandri, 2008]. Myostatin, a member of the TGF $\beta$ family predominantly expressed in skeletal muscle, conversely functions as a potent cytokine for the inhibition of muscle growth and induction of muscle atrophy. Following activation and release from the ECM, myostatin activates Smad2/3 and TAK1/p38 MAPK signaling cascades, leading to the upregulation of atrogin- 1 and muscle ring-finger protein (MuRF-1), and subsequent proteolysis [Gumucio and Mendias, 2013]. Both IGF-1 and myostatin, along with their downstream effectors, have been targeted for therapeutic use in vivo [Sandri, 2008; Zanou and Gailly, 2013], and it is expected that similar benefits will translate to engineered skeletal muscle. External to the IGF/Akt/ mTOR signaling pathway, several growth factors have been examined due to their effects on myogenic progenitor cells implicated in skeletal muscle growth and development. As in embryonic development, TGF $\beta$ inhibits progression of muscle precursor cells [Wagers and Conboy, 2005; Sandri, 2008]. In contrast, HGF, FGF, and platelet-derived growth factor (PDGF) have all been associated with muscle repair and hypertrophy [Husmann et al., 1996; Huard et al., 2002]. As in postnatal development, HGF is released from the ECM with injury to

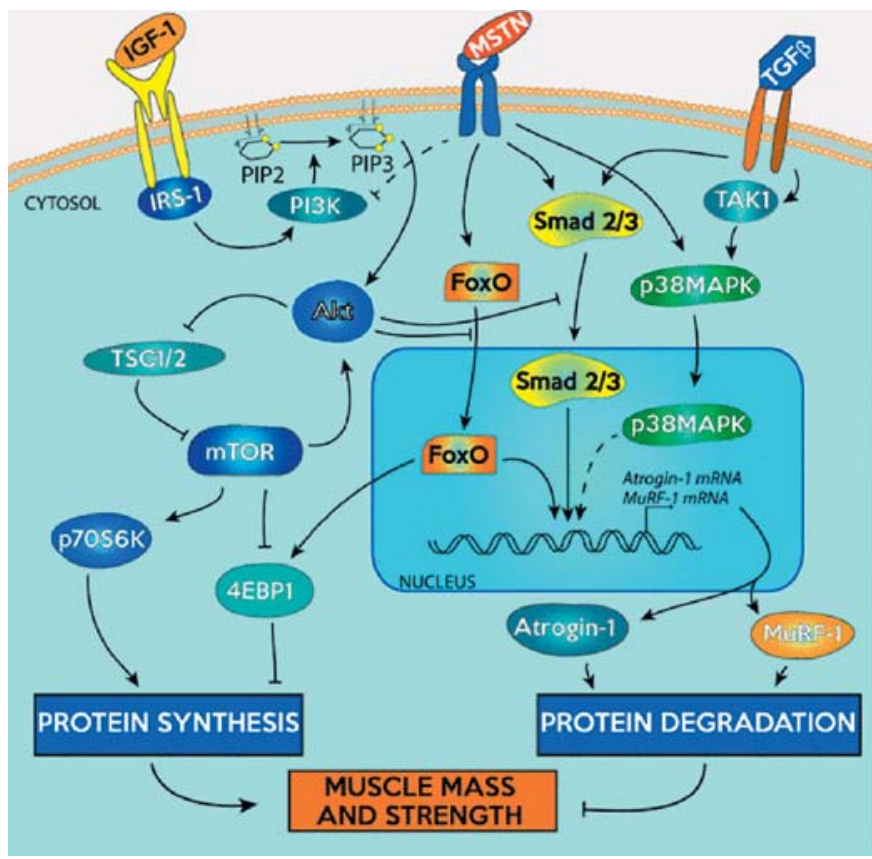

Fig. 3. Central pathways to the regulation of skeletal muscle hypertrophy and atrophy. Adapted from Gumucio and Mendias [2013].

prompt satellite cell activation and proliferation via p38 MAPK and PI3K [Zanou and Gailly, 2013]. FGF serves a similar purpose, mainly stimulating proliferation of satellite cells. After release from activated platelets and macrophages, $\mathrm{PDGF}$ again promotes myogenic proliferation in addition to angiogenesis. Interestingly, these three growth factors (HGF, FGF, and PDGF) all have an inhibitory effect on myogenic differentiation [Husmann et al., 1996; Zanou and Gailly, 2013]. Overall, it is evident how various growth factors are intricately involved in these native pathways controlling the development, growth, and regeneration of skeletal muscle. Their interplay with the canonical myogenic regulatory factors is evident in the activation and proliferation of myogenic progenitors, terminal differentiation to myoblasts, and up- or downregulation of protein synthesis and degradation.

\section{Growth Factors for Skeletal Muscle Tissue Engineering}

Tissue engineers have used this understanding of growth factors and their role in myogenesis to direct techniques for the fabrication of skeletal muscle. To 
date, tissue engineering technologies utilize either scaffold materials ranging from decellularized tissues [ $\mathrm{Wu}$ et al., 2012; Sicari et al., 2014] to collagen and fibrin hydrogels [Lam et al., 2009; Lee and Vandenburgh, 2013; Juhas et al., 2014] or opt for a scaffold-free approach [Carosio et al., 2013; Williams et al., 2013; VanDusen et al., 2014] to promote the development of ECM for subsequent muscle tissue. The success of these techniques ultimately depends on the in vitro cultivation of isolated primary muscle precursor cells for the development of mature muscle cells within the ECM. The typical techniques for culture of these myogenic cells involves an initial proliferation phase, to allow cell numbers to expand to a sufficiently large population, followed by differentiation and fusion into myotubes and maturation to myofibers [Clegg et al., 1987]. Detailed study of growth factors known to play a role in each of these phases of myogenesis is thus essential to further the understanding of muscle growth in vitro. It is generally accepted that media rich in serum promote initial proliferation of skeletal muscle stem cells to myoblasts while delaying the onset of differentiation, potentially due to a large number of hormones and growth factors of varying concentration and potency found in the serum [Allen et al., 1997]. Dramatic changes in function and speed of contraction were recently observed between engineered muscles cultured in serum from the United States and from the European Union [Khodabukus and Baar, 2014]. Similarly, environmental factors in the culture media, such as glucose and antibiotic concentration, have been shown to alter engineered muscle phenotype and function [Khodabukus and Baar, 2015]. In this study, ideal conditions for maximizing force production in tissue engineered skeletal muscle involved high glucose (25 $\mathrm{mM}$ ) and absence of streptomycin. A drastically reduced serum content in media following the proliferation phase triggers differentiation of the myoblast to the myotube, possibly due to the absence of key mitogenic components. Due to the lot-to-lot variations in growth factors present in the commercially available serum and the consequent variability in satellite cell induction and proliferation, an optimum serum formulation and the identity of these mitogenic components has yet to be fully defined [Doumit and Merkel, 1992]. To date, several growth factors have been implicated as potential serum components with important myogenic effects.

As would be expected based on their in vivo influence on skeletal muscle hypertrophy described above, FGF, PDGF, and HGF promote activation and proliferation of myogenic progenitor cells and delay terminal differen- tiation [Allen et al., 1995; Husmann et al., 1996; Kuang et al., 2008]. The stimulatory effect of FGF on satellite cell proliferation has been shown to produce a twofold increase in DNA content relative to untreated cultures [Düsterhöft and Pette, 1999]. This enhanced proliferation resulted in the formation of larger myotubes and increased expression of myogenin and MHC. When added during the differentiation phase of the culture, however, these significant effects were not observed [Maley et al., 1995; Düsterhöft and Pette, 1999]. Similarly, the addition of PDGF led to a twofold increase in DNA synthesis and improved satellite cell proliferation, but did not yield a significant increase in desmin expression and myotube formation [Yablonka-Reuveni et al., 1990; Maley et al., 1995]. In the case of HGF, the time between the isolation of satellite cells and the onset of the cell cycle decreased from approximately $42-60$ to $<24 \mathrm{~h}$ [Allen et al., 1995]. These results demonstrate conservation of the signaling pathways involved in native myogenesis in an in vitro setting, with HGF activating quiescent satellite cells to begin progressing down the myogenic lineage. By combining HGF with either FGF or PDGF, tissue engineers may be able to maximize the replicative potential of satellite cells by achieving activation earlier and then increasing subsequent proliferation. Furthermore, HGF and FGF have a tendency to delay terminal differentiation, allowing for extension of the window for satellite cell proliferation. Additionally, IGF plays a key role in all phases of satellite cell myogenesis, from activation and proliferation to induction of the onset of myogenic differentiation [Allen and Boxhorn, 1989; Chakravarthy et al., 2000]. In one study, IGF was supplied in the fibrin gel used as a 3D scaffold to support the fabricated muscle tissue [Huang et al., 2004]. The beneficial effects of IGF addition were seen in the form of MyoD upregulation during initial proliferation, followed by a $50 \%$ increase in force production in the final engineered tissue. The functional improvement with IGF addition illustrates its importance in engineering skeletal muscle. In contrast, TGF $\beta$ typically has a negative influence on both myogenic phases in vitro, slightly suppressing proliferation and severely inhibiting differentiation [Allen and Boxhorn, 1989; Maley et al., 1995; Husmann et al., 1996]. TGF $\beta$, however, can enhance contractility of engineered muscle by promoting collagen type I synthesis in the ECM, supporting myofiber development and force transmission [Weist et al., 2013]. As a result of these findings, it is common to supplement media supplied during the initial proliferation phase with HGF and FGF prior to switching to a medium sup- 


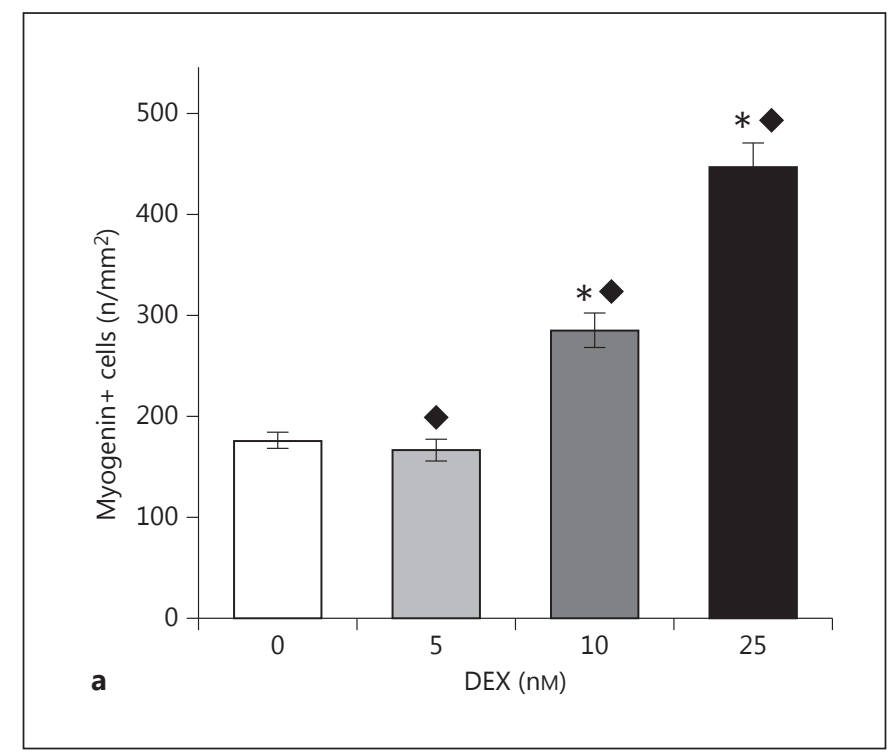

Fig. 4. DEX effects on myogenic differentiation. a Myogenin expression indicated increasing terminal differentiation of satellite cells and muscle progenitors into myoblasts. A dose-dependent response to DEX addition was observed for myogenin-positive cell density. b Myotube fusion calculated from desmin and DAPI

plemented with IGF or insulin for the induction of differentiation [Lee and Vandenburgh, 2013; Williams et al., 2013]. By adding these specific growth factors to influence satellite cell proliferation and differentiation, tissue engineers have successfully created skeletal muscle constructs in vitro featuring neonatal functional and structural characteristics [Williams et al., 2013; VanDusen et al., 2014].

\section{Effects of DEX on Satellite Cells during Skeletal Muscle Tissue Engineering}

DEX has previously been used to influence satellite cell cultures in vitro, but its potential benefits for tissue engineered skeletal muscle have yet to be examined. To expand on the understanding of DEX during fabrication of tissue engineered skeletal muscle, our laboratory has studied the effect of dose and time of administration of DEX on our engineered muscle constructs. Clinically, DEX has anti-inflammatory or immunosuppressant activity and is used in treating several rheumatologic and skin diseases, in addition to severe asthma and allergies. In skeletal muscle, exogenously delivered DEX has profoundly different effects depending on the dosage and

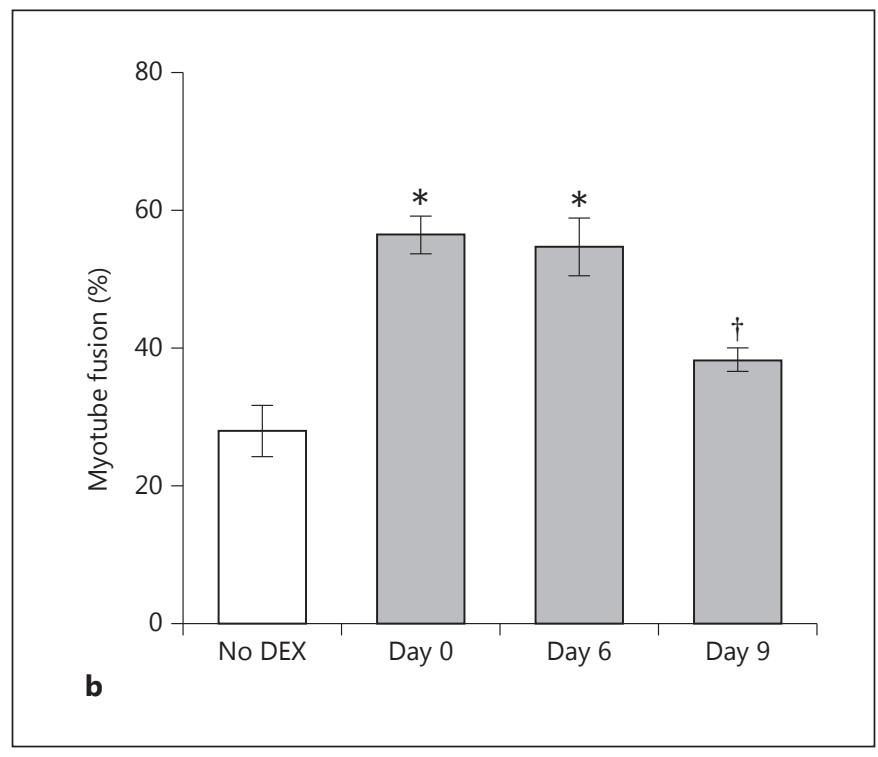

staining quantified the number of nuclei associated with a myotube as a percentage of total nuclei and was significantly increased with DEX addition on day 0 and day 6 . Means \pm SE. Statistically significant differences vs. control $(*)$, other DEX concentrations $(\diamond)$, and other DEX timings $(\dagger)$ are indicated. timing of the administration. When administered at supraphysiological doses to adult skeletal muscle, DEX leads to atrophy through upregulation of the myostatin promoter and inhibition of IGF-1 expression (fig. 3) [Inder et al., 2010; Qin et al., 2013]. These findings make it a potential agent for the induction of adult skeletal muscle myopathy. In contrast, the addition of 5-25 nM DEX has been shown to improve myogenesis in vitro by enhancing differentiation and myotube fusion, potentially through its induction of dysferlin, a calcium-binding transmembrane protein thought to play an important role in both myogenesis and membrane repair [Belanto et al., 2010]. Other in vitro studies have demonstrated that DEX can inhibit myoblast proliferation and protein synthesis [Desler et al., 1996], however, reinforcing the need for careful timing of its addition to the culture. The studies referenced above, delineating the effects of DEX in vitro, primarily experimented with the immortal C2C12 mouse myoblast cell line rather than the primary cell population used in our tissue engineering methods. We exposed the heterogeneous pool of cells obtained from soleus muscle isolation (primarily satellite cells and fibroblasts, but also endothelial cells, perivascular cells, immune cells, and several other cell types) to different doses of DEX. By administering DEX at several 
time points, we found the optimal conditions for improving myogenesis, ultimately maximizing in vitro structural and functional development of tissue engineered skeletal muscle. These findings are briefly summarized below.

\section{Effect of DEX on Muscle Satellite Cell Differentiation} and Myotube Fusion

Using our established SMU fabrication protocol, muscle isolates were cultured at four DEX concentrations ( 0 , 5, 10, and $25 \mathrm{nM}$ ) [Syverud et al., 2016]. Following seeding onto a laminin-coated Sylgard substrate, the administration of DEX was initiated concomitant with seeding (day 0 ), during the proliferative stage (day 6), or in the differentiation phase (day 9), and was sustained until the completion of SMU fabrication. Immunocytochemical analysis of myogenin expression was used as an indicator of satellite cell differentiation into myoblasts [McFarland et al., 2013]. A dose-dependent increase in myogenin-positive cell density was observed in response to DEX administration (fig. 4a) regardless of the growth stage at which it was added.

Progressing along the myogenic pathway, the myotube fusion percentage was analyzed to assess the ability of myoblasts to form a robust network of terminally differentiated myotubes in the presence of DEX. All three doses of DEX $(5,10$, and $25 \mathrm{nM})$ significantly improved myotube fusion compared to untreated controls (no DEX). No significant difference was observed between the three doses. Early addition of DEX at either cell seeding on day 0 or during the proliferative stage on day 6 resulted in significantly increased myotube fusion (fig. 4b). Treatment with DEX during the subsequent differentiation stage (day 9), however, resulted in no improvement in myotube fusion; in fact, there was no difference compared to controls.

In addition to myotube fusion, myotube size and number were also affected by the administration of DEX at early time points. Again, DEX addition during either cell seeding (day 0 ) or the proliferative stage (day 6) significantly increased myotube diameter (fig. 5). Treatment with DEX during the later differentiation stage (day 9) did not improve myotube diameter. In addition, a dose-dependent response to DEX was observed, with myotube diameter consistently increasing as DEX concentration increased. Not surprisingly, the greatest effect of DEX on myotube density occurred when DEX was administered during the proliferative stage (day 6). Administration of DEX on either day 0 or during differentiation (day 9) showed no effect and resulted in cell densities similar to the untreated controls. Additionally, no differences in myotube densities were observed with respect to DEX concentrations. Together, myotube size and density data suggest that addition of DEX on day 6 leads to an increased number of more robust myotubes, which may be preferable for engineering skeletal muscle.

\section{Structural and Functional Maturation of SMUs with Addition of DEX}

Following 3D formation, our SMUs were evaluated for function by assessing contractile force production. The isometric tetanic forces produced by engineered SMUs are displayed in figure $5 \mathrm{c}$. Interestingly, only the $10 \mathrm{nM}$ DEX concentration led to an improvement in function, characterized by a fivefold increase in the maximal isometric force production compared to no-DEX controls. The SMUs that received $5 \mathrm{nM}$ DEX did not show any improvement in force production compared to control SMUs. Furthermore, the addition of $25 \mathrm{nM}$ DEX led to a significant decrease in force production compared to controls. This decrease in function at the 25-nM DEX dosage may have resulted from cell injury, indicated by the formation of blebs in the periphery of several myotubes [Wang et al., 2010]. Alternatively, since DEX can potentially upregulate fibroblast proliferation and tissue fibrosis [Dammeier et al., 1998], it is possible that the 25-nM dosage may have prompted fibroblast overgrowth at the expense of engineered muscle function. Again, the early addition of DEX during either cell seeding (day 0 ) or during the proliferative stage (day 6) resulted in the greatest improvement in force production. In support of the increased force production observed following early-phase administration of $10 \mathrm{nM}$ DEX, immunocytochemical analysis of $\alpha$-actinin showed advanced maturation of myotubes demonstrated by the presence of the advanced sarcomeric structure within highly aligned myofibrils (fig. 5). In contrast, no sarcomeric structure was observed in the control muscle cultures.

\section{Current Understanding of Growth Factors and DEX in Skeletal Muscle Tissue Engineering}

The overall effects of DEX during the SMU fabrication process can be divided into differentiation and maturation phases. In agreement with previous findings in the literature [Belanto et al., 2010], addition of DEX at 10 and $25 \mathrm{~nm}$ led to increased myogenic differentiation into myoblasts, as shown by the expression of the canonical myogenic regulatory factor, myogenin, followed 

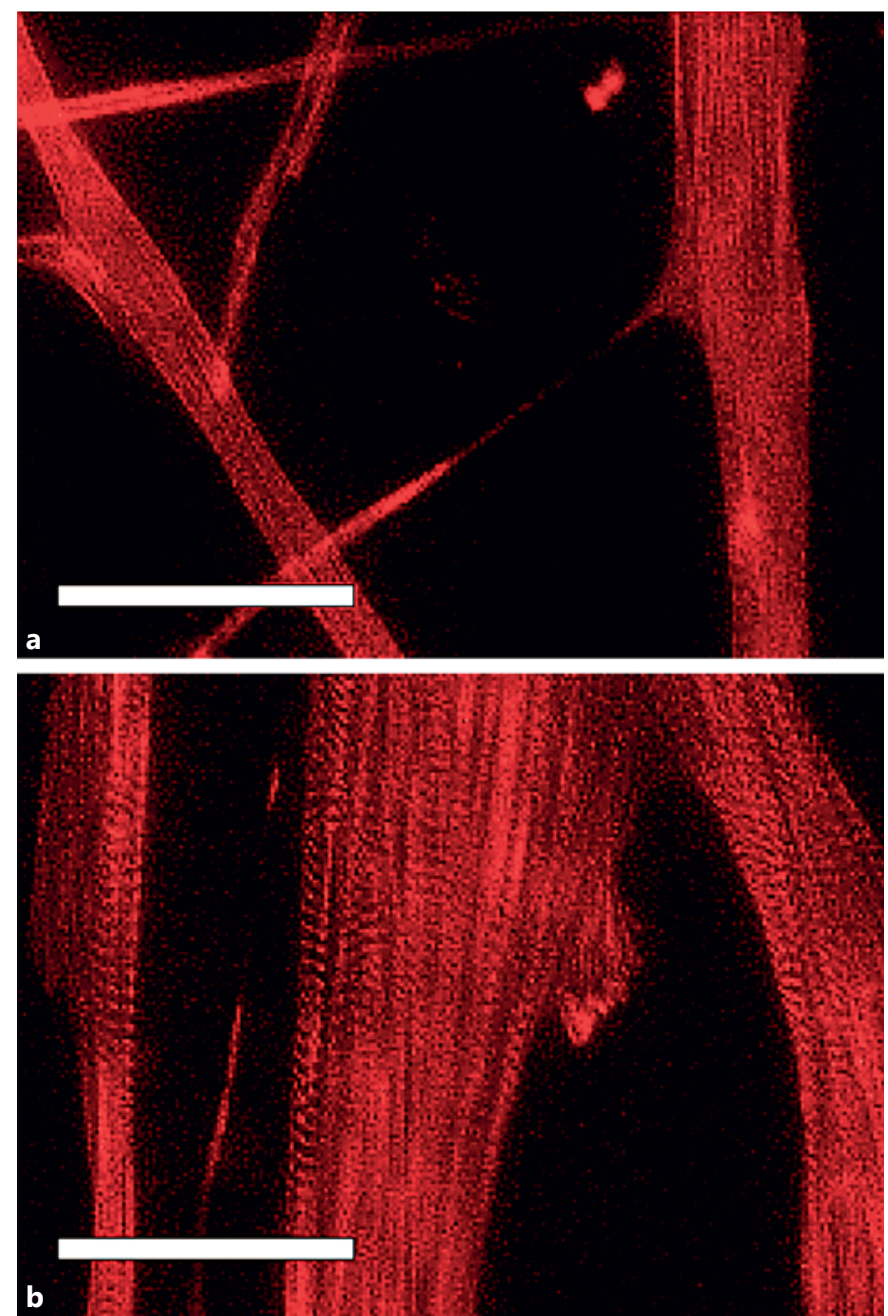

by eventual fusion into myotubes. Generally, the 25-nM concentration led to greater differentiation and fusion, but, interestingly, those increases did not translate into improved force production. Overall, this experiment demonstrated that the addition of exogenous DEX to isolated muscle satellite cells can improve force production and structural characteristics of our tissue engineered skeletal muscle when administered at optimal doses and timings. The most promising results were achieved with the addition of $10 \mathrm{nM}$ DEX on either day 0 or day 6 . In addition to improved myogenic differentiation and myotube fusion, SMUs exposed to this concentration exhibited substantially accelerated structural maturation accompanied by a fivefold increase in force production.

These DEX experiments were conducted as a means of supplementing our existing protocol for engineering

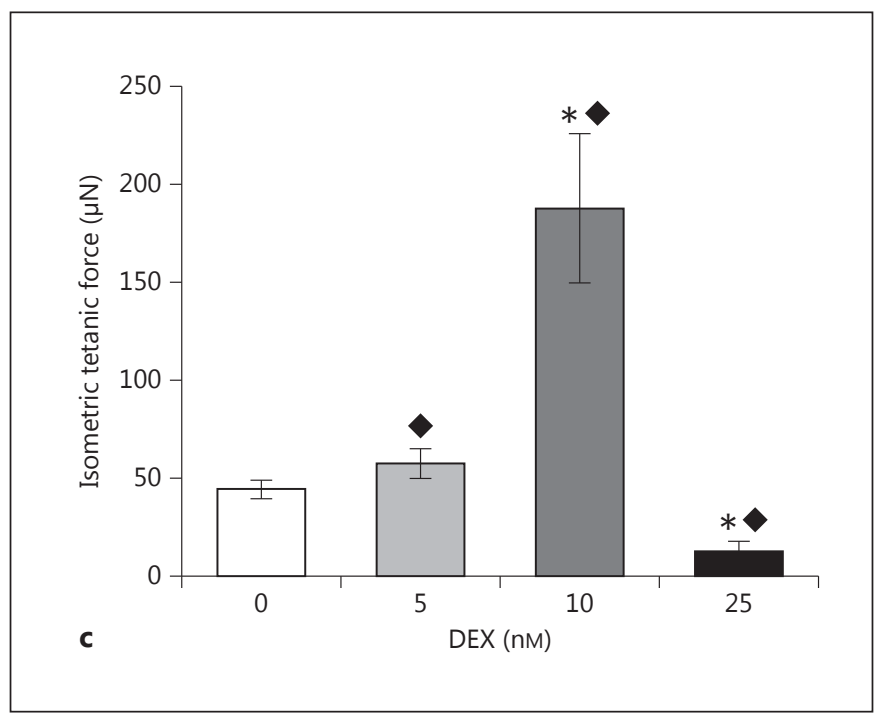

Fig. 5. SMU maturation with DEX addition. Representative images of engineered skeletal muscle without DEX (a) and following DEX addition (b). Images show $\alpha$-actinin in developing muscle monolayers just 10 days after seeding. Formation of advanced sarcomeric structure and aligned myofibrils was evident in DEXtreated plates at this early time point. a, b Scale bars $=50 \mu \mathrm{m}$. c Based on functional measures of isometric tetanic force in DEXtreated SMUs, the addition of $10 \mathrm{nM} \mathrm{DEX}$ is optimal for SMU fabrication. Interestingly, the addition of $25 \mathrm{nM}$ DEX had a consistently detrimental effect on force production. Means \pm SE. Statistically significant differences vs. control $(*)$ and other DEX concentrations $(\bullet)$ are indicated. skeletal muscle. This established SMU fabrication protocol [VanDusen et al., 2014] has capitalized on the advances in satellite cell cultures and skeletal muscle tissue engineering described in the introduction. Specifically, FGF is supplied in the growth media as a means of maximizing satellite cell proliferation and delaying terminal differentiation, serving a role similar to either PDGF or HGF during this phase. Insulin is subsequently added to differentiation media as an analog to IGF for induction of myogenic differentiation. IGF acts as a potent inducer of myogenesis, both in vivo and in vitro, and is the only growth factor known to promote both satellite cell proliferation and differentiation. By using a novel application of DEX to build on our existing tissue engineering expertise, this article presents a blueprint for advancing tissue engineering of skeletal muscle. 


\section{Acknowledgments}

The authors would like to acknowledge the support of the NIH R56 grant 2-R56-AR-054778-06-A1 and the Microfluidics in Biomedical Sciences Training Program NIH NIBIB T32 EB005582.

\section{Disclosure Statement}

The authors have nothing to disclose.

\section{References}

Allen, R.E., L.K. Boxhorn (1989) Regulation of skeletal muscle satellite cell proliferation and differentiation by transforming growth factor-beta, insulin-like growth factor I, and fibroblast growth factor. J Cell Physiol 138: 311-315.

Allen, R.E., S.M. Sheehan, R.G. Taylor, T.L. Kendall, G.M. Rice (1995) Hepatocyte growth factor activates quiescent skeletal muscle satellite cells in vitro. J Cell Physiol 165: 307-312.

-Allen, R.E., C.J. Temm-Grove, S.M. Sheehan, G. Rice (1997) Skeletal muscle satellite cell cultures. Methods Cell Biol 52: 155-176.

Bach, A.D., J. Stern-Straeter, J.P. Beier, H. Bannasch, G.B. Stark (2003) Engineering of muscle tissue. Clin Plast Surg 30: 589-599.

Belanto, J.J., S.V. Diaz-Perez, C.E. Magyar, M.M. Maxwell, Y. Yilmaz, K. Topp, G. Boso, C.H. Jamieson, C.A.M. Jamieson, N.A. Cacalano (2010) Dexamethasone induces dysferlin in myoblasts and enhances their myogenic differentiation. Neuromuscul Disord 20: 111121.

Bentzinger, C.F., Y.X. Wang, M.A. Rudnicki (2012) Building muscle: molecular regulation of myogenesis. Cold Spring Harb Perspect Biol 4: a008342.

Biressi, S., M. Molinaro, G. Cossu (2007) Cellular heterogeneity during vertebrate skeletal muscle development. Dev Biol 308: 281-293.

Buckingham, M., A. Mayeuf (2012) Skeletal muscle development; in Olson, J.A.H.N. (ed): Muscle. Boston/Waltham, Academic Press, chapt 52, pp 749-762.

Carosio, S., L. Barberi, E. Rizzuto, C. Nicoletti, Z. Del Prete, A. Musarò (2013) Generation of eX vivo-vascularized muscle engineered tissue (X-MET). Sci Rep 3: 1420-1429.

-Chakravarthy, M.V., T.W. Abraha, R.J. Schwartz, M.L. Fiorotto, F.W. Booth (2000) Insulin-like growth factor-I extends in vitro replicative life span of skeletal muscle satellite cells by enhancing G1/S cell cycle progression via the activation of phosphatidylinositol $3^{\prime}$-kinase/ Akt signaling pathway. J Biol Chem 275: 35942-35952.

Clegg, C.H., T.A. Linkhart, B.B. Olwin, S.D. Hauschka (1987) Growth factor control of skeletal muscle differentiation: commitment to terminal differentiation occurs in G1 phase and is repressed by fibroblast growth factor. J Cell Biol 105: 949-956.

Corona, B.T., C.L. Ward, H.B. Baker, T.J. Walters, G.J. Christ (2014) Implantation of in vitro tissue engineered muscle repair constructs and bladder acellular matrices partially restore in vivo skeletal muscle function in a rat model of volumetric muscle loss injury. Tissue Eng Part A 20: 705-715.

Cosgrove, B.D., A. Sacco, P.M. Gilbert, H.M. Blau (2009) A home away from home: challenges and opportunities in engineering in vitro muscle satellite cell niches. Differentiation 78: 185-194.

Dammeier, J., H.D. Beer, M. Brauchle, S. Werner (1998) Dexamethasone is a novel potent inducer of connective tissue growth factor expression - implications for glucocorticoid therapy. J Biol Chem 273: 18185-18190.

Dennis, R.G., B. Smith, A. Philp, K. Donnelly, K. Baar (2009) Bioreactors for guiding muscle tissue growth and development. Adv Biochem Engin Biotechnol 112: 39-79.

Desler, M.M., S.J. Jones, C.W. Smith, T.L. Woods (1996) Effects of dexamethasone and anabolic agents on proliferation and protein synthesis and degradation in $\mathrm{C} 2 \mathrm{C} 12$ myogenic cells. J Anim Sci 74: 1265-1273.

Dhawan, J., T.A. Rando (2005) Stem cells in postnatal myogenesis: molecular mechanisms of satellite cell quiescence, activation and replenishment. Trends Cell Biol 15: 666-673.

Doumit, M.E., R.A. Merkel (1992) Conditions for isolation and culture of porcine myogenic satellite cells. Tissue Cell 24: 253-262.

Düsterhöft, S., D. Pette (1999) Evidence that acidic fibroblast growth factor promotes maturation of rat satellite-cell-derived myotubes in vitro. Differentiation 65: 161-169.

Glass, D.J. (2003) Signalling pathways that mediate skeletal muscle hypertrophy and atrophy. Nat Cell Biol 5: 87-90.

-Glass, D.J. (2005) Skeletal muscle hypertrophy and atrophy signaling pathways. Int J Biochem Cell Biol 37: 1974-1984.

Grefte, S., A.M. Kuijpers-Jagtman, R. Torensma, J.W.V.d. Hoff (2007) Skeletal muscle development and regeneration. Stem Cells Dev 16: 857-868.

Gumucio, J. P., C.L. Mendias (2013) Atrogin-1, MuRF-1, and sarcopenia. Endocrine 43: 12-21.

Huang, Y., R.G. Dennis, L.M. Larkin, K. Baar (2004) Rapid formation of functional muscle in vitro using fibrin gels. J Appl Physiol 98: 706-713.

Huard, J., Y. Li, F.H. Fu (2002) Muscle injuries and repair: current trends in research. J Bone Joint Surg 84-A: 822-832.

Husmann, I., L. Soulet, J. Gautron, I. Martelly, D. Barritault (1996) Growth factors in skeletal muscle regeneration. Cytokine Growth Factor $\operatorname{Rev} 7: 249-258$.
Inder, W.J., C. Jang, V.R. Obeyesekere, F.P. Alford (2010) Dexamethasone administration inhibits skeletal muscle expression of the androgen receptor and IGF-1 - implications for steroid-induced myopathy. Clin Endocrinol (Oxf) 73: 126-132.

Jackman, R.W., S.C. Kandarian (2004) The molecular basis of skeletal muscle atrophy. Am J Physiol Cell Physiol 287: 834-843.

Juhas, M., G.C. Engelmayr, A.N. Fontanella, G.M. Palmer, N. Bursac (2014) Biomimetic engineered muscle with capacity for vascular integration and functional maturation in vivo. Proc Natl Acad Sci USA 111: 5508-5513.

Khodabukus, A., K. Baar (2014) The effect of serum origin on tissue engineered skeletal muscle function. J Cell Biochem 115: 2198.

Khodabukus, A., K. Baar (2015) Glucose concentration and streptomycin alter in vitro muscle function and metabolism. J Cell Physiol 230: 1226.

Koning, M., M.C. Harmsen, M.J.A. van Luyn, P.M.N. Werker (2009) Current opportunities and challenges in skeletal muscle tissue engineering. J Tissue Eng Regen Med 3: 407-415.

Kuang, S., M.A. Gillespie, M.A. Rudnicki (2008) Niche regulation of muscle satellite cell selfrenewal and differentiation. Cell Stem Cell 2: 22-31.

Lam, M.T., Y. Huang, R.K. Birla, S. Takayama (2009) Microfeature guided skeletal muscle tissue engineering for highly organized 3-dimensional free-standing constructs. Biomaterials 30: 1150-1155.

- Lee, P.H.U., H.H. Vandenburgh (2013) Skeletal muscle atrophy in bioengineered skeletal muscle: a new model system. Tissue Eng Part A 19: 2147-2155.

Maley, M.A.L., M.J. Davies, M.D. Grounds (1995) Extracellular matrix, growth factors, genetics: their influence on cell proliferation and myotube formation in primary cultures of adult mouse skeletal muscle. Exp Cell Res 219: 169179.

Martin, N.R.W., S.L. Passey, D.J. Player, A. Khodabukus, R.A. Ferguson, A.P. Sharples, V. Mudera, K. Baar, M.P. Lewis (2013) Factors affecting the structure and maturation of human tissue engineered skeletal muscle. Biomaterials 34: 5759-5765.

Mathew, S.J., J.M. Hansen, A.J. Merrell, M.M. Murphy, J.A. Lawson, D.A. Hutcheson, M.S. Hansen, M. Angus-Hill, G. Kardon (2011) Connective tissue fibroblasts and Tcf4 regulate myogenesis. Development 138: $371-$ 384. 
Mauro, A. (1961) Satellite cell of skeletal muscle fibers. J Biophys Biochem Cytol 9: 493-495.

McFarland, D.C., J.E. Pesall, C.S. Coy, S.G. Velleman (2013) Effects of 17ß-estradiol on turkey myogenic satellite cell proliferation, differentiation, and expression of glypican-1, MyoD and myogenin. Comp Biochem Physiol A Mol Integr Physiol 164: 565-571.

Murphy, M.M., J.A. Lawson, S.J. Mathew, D.A. Hutcheson, G. Kardon (2011) Satellite cells, connective tissue fibroblasts and their interactions are crucial for muscle regeneration. Development 138: 3625-3637.

Neal, D., M.S. Sakar, L.S. Ong, H.H. Asada (2014) Formation of elongated fascicle-inspired 3D tissues consisting of high-density, aligned cells using sacrificial outer molding. Lab Chip 14: 1907-1916.

Qin, J., R. Du, Y. Yang, H. Zhang, Q. Li, L. Liu, H. Guan, J. Hou, X. An (2013) Dexamethasoneinduced skeletal muscle atrophy was associated with upregulation of myostatin promoter activity. Res Vet Sci 94: 84-89.

-Sakar, M.S., D. Neal, T. Boudou, M.A. Borochin, Y. Li, R. Weiss, R.D. Kamm, C.S. Chen, H.H. Asada (2012) Formation and optogenetic control of engineered 3D skeletal muscle bioactuators. Lab Chip 12: 4976.

-Sandri, M. (2008) Signaling in muscle atrophy and hypertrophy. Physiology 23: 160-170.

-Schiaffino, S., C. Mammucari (2011) Regulation of skeletal muscle growth by the IGF1-Akt/ PKB pathway: insights from genetic models. Skelet Muscle 1: 4.
Sicari, B.M., J.P. Rubin, C.L. Dearth, M.T. Wolf, F. Ambrosio, M. Boninger, N.J. Turner, D.J. Weber, T.W. Simpson, A. Wyse, E.H.P. Brown, J.L. Dziki, L.E. Fisher, S. Brown, S.F. Badylak (2014) An acellular biologic scaffold promotes skeletal muscle formation in mice and humans with volumetric muscle loss. Sci Transl Med 6: 234ra58.

Syverud, B., K. VanDusen, L. Larkin (2016) Effects of dexamethasone on satellite cells and tissue engineered skeletal muscle units. Tissue Eng Part A 22: 480-489.

VanDusen, K.W., B.C. Syverud, M.L. Williams, J.D. Lee, L.M. Larkin (2014) Engineered skeletal muscle units for repair of volumetric muscle loss in the tibialis anterior muscle of a rat. Tissue Eng Part A 20: 2920-2930.

Wagers, A.J., I.M. Conboy (2005) Cellular and molecular signatures of muscle regeneration: current concepts and controversies in adult myogenesis. Cell 122: 659-667.

Wang, B., Z. Yang, B.K. Brisson, H. Feng, Z. Zhang, E.M. Welch, S.W. Peltz, E.R. Barton, R.H. Brown, Jr., H.L. Sweeney (2010) Membrane blebbing as an assessment of functional rescue of dysferlin-deficient human myotubes via nonsense suppression. J Appl Physiol 109: 901-905.
Weist, M.R., M.S. Wellington, J.E. Bermudez, T.Y. Kostrominova, C.L. Mendias, E.M. Arruda, L.M. Larkin (2013) TGF $\beta 1$ enhances contractility in engineered skeletal muscle. J Tissue Eng Regen Med 7: 562-571.

Williams, M.L., T.Y. Kostrominova, E.M. Arruda, L.M. Larkin (2013) Effect of implantation on engineered skeletal muscle constructs. J Tissue Eng Regen Med 7: 434-442.

Wu, X., B.T. Corona, X. Chen, T.J. Walters (2012) A standardized rat model of volumetric muscle loss injury for the development of tissue engineering therapies. BioRes Open Access 1: 280-290.

Yablonka-Reuveni, Z., T.M. Balestreri, D.F. Bowen-Pope (1990) Regulation of proliferation and differentiation of myoblasts derived from adult mouse skeletal muscle by specific isoforms of PDGF. J Cell Biol 111: 1623-1629.

$\checkmark$ Yin, H., F. Price, M.A. Rudnicki (2013) Satellite cells and the muscle stem cell niche. Physiol Rev 93: 23-67.

Zanou, N., P. Gailly (2013) Skeletal muscle hypertrophy and regeneration: interplay between the myogenic regulatory factors (MRFs) and insulin-like growth factors (IGFs) pathways. Cell Mol Life Sci 70: 4117.

Zhang, P., X. Liang, T. Shan, Q. Jiang, C. Deng, R. Zheng, S. Kuang (2015) mTOR is necessary for proper satellite cell activity and skeletal muscle regeneration. Biochem Biophys Res Commun 463: 102. 